\title{
Assessment of systemic inflammation with neutrophil-lymphocyte ratio in lichen planus
}

\author{
Hatice Ataş, Bengü Çevirgen Cemil, Gökçe Işıl Kurmuş, Müzeyyen Gönül
}

Department of Dermatology, Dışkapı Yıldııım Beyazıt Training and Research Hospital, Ankara, Turkey

Adv Dermatol Allergol 2016; XXXIII (3): 188-192

DOI: 10.5114/pdia.2016.56930

\begin{abstract}
Introduction: Lichen planus (LP) is a papulosquamous eruption of the skin and mucous membranes. Although the exact pathogenesis of the disease remains unclear, it is believed that LP represents an inflammatory disorder. Neutrophil-lymphocyte $(\mathrm{N} / \mathrm{L})$ ratio is considered a systemic inflammatory marker that correlated with severity of the diseases.

Aim: To investigate whether N/L ratio increases in LP and may be an independent severity marker for LP lesions. Material and methods: White blood cell (WBC), neutrophil and lymphocyte counts, N/L ratio, erythrocyte sedimentation rate (ESR) and C-reactive protein (CRP) were statistically compared between the patient $(n=55)$ and the control group $(n=48)$. The relationship of N/L ratio and the body surface area (BSA) was assessed.

Results: Erythrocyte sedimentation rate and CRP were statistically higher in patients with LP than in controls $(p<0.0001)$. Our analysis revealed a significantly higher level of $\mathrm{N} / \mathrm{L}$ ratio in patients with LP compared with controls, respectively $(2.5 \pm 1.1(1.2-7.3)$ vs. $1.4 \pm 0.4(0.8-2.7), p<0.0001)$. Body surface area $(p=0.001)$, CRP $(p=0.006)$, and $\operatorname{ESR}(p=0.003)$ were identified as possible predictors of N/L ratio, but only BSA $(p=0.002)$ and $\operatorname{ESR}(p=0.003)$ were found as significant independent predictors in a multiple linear regression model.

Conclusions: The inflammatory process in LP was supported by our results. N/L ratio may have an impact to show the inflammatory status in patients with LP as an inexpensive, simple and effective predictor. It may be used for the severity and treatment option of LP. But, N/L ratio and LP relationship could be confirmed by other large prospective studies.
\end{abstract}

Key words: systemic, inflammation, neutrophil lymphocyte ratio, lichen planus.

\section{Introduction}

Lichen planus (LP) is an uncommon skin and/or mucous membrane disorder that is assumed to be closely related to dyslipidemia and diabetes mellitus [1, 2]. In a case-control study, patients with lichen planus were found to have increased both metabolic and cardiovascular disorders in relation to controls, most probably due to long standing inflammation [3]. These findings support that LP is not only a chronic inflammatory disease restricted to the skin, but also a systemic one.

Biochemical and hematologic markers can help us show systemic inflammation; however, most of these markers are usually expensive and ineffective. Neutrophil-lymphocyte $(\mathrm{N} / \mathrm{L})$ ratio is considered a systemic inflammatory marker. There is a significant association between systemic inflammation measured by N/L ratio and prevalent chronic conditions [4]. The relationship between severity of disease and increased inflammatory response are supported in psoriasis and Behcet's disease $[5,6]$. Neutrophil-lymphocyte ratio has not been evaluated in patients with LP yet.

\section{Aim}

In this study, we aim to investigate whether there is an inflammatory process in patients with LP, and clinical usefulness of $\mathrm{N} / \mathrm{L}$ ratio which is considered and supported as a systemic inflammatory marker. 


\section{Material and methods}

\section{Subjects}

This is a single-center and case-control study of 101 subjects (patients with LP $=55$, controls $=48$ ) who were admitted to the Department of Dermatology at Diskapi Yildirim Beyazit Training Hospital between January 2013 and July 2014. They were randomly selected from the dermatology clinic. Controls were randomly selected from healthy individuals or patients who were admitted to the dermatology clinic for minimal dermatological problems such as nevus in order to avoid bias to the study results. Approval for the study was obtained from the Institution Ethics Committee. Informed written consent was obtained from subjects.

Inclusion criteria for the study group were as follows: to be a newly diagnosed LP patient clinically and/or histopathologically, not undergoing any treatment for LP before the study, age older than 18 years, and willingness to give informed consent to participate in the study.

Exclusion criteria were as follows: patients suffering from chronic diseases such as hepato-renal disease, diabetes mellitus, cancer, allergic disease, thyroid dysfunction, taking some medicines or drugs affecting laboratory parameters, any drug therapy which may cause LP-like lesions, epithelial dysplasia and inflammatory or infection diseases in patients and controls.

Clinical data were recorded for each subject including age, sex, duration of disease, involvement site and body surface aera (BSA), C-reactive protein (CRP), white blood cells (WBC), neutrophil and lymphocyte counts, erythrocyte sedimentation rate (ESR). In controls, we evaluated CBC count, CRP, and ESR. We have considered the BSA as the severity of the disease.

\section{Biochemical analyses}

Blood samples were obtained by venipuncture. Complete blood counts were measured by the method of laser-based optic calorimetric, using an automated blood cell counter (Coulter LH 750 analyzer; Beckman Coulter, Galway, Ireland). N/L ratio was estimated as the ratio of the neutrophil to-lymphocyte counts. An automated erythrocyte sedimentation rate analyzer which is compatible with Westergreen method was used for ESR (0$20 \mathrm{~mm} / \mathrm{h}$ ). C-reactive protein reaction ( $<1 \mathrm{mg} / \mathrm{dl}$ ) levels were measured using the immunoturbidimetric method.

\section{Statistical analysis}

Statistical analyses were performed using the SPSS software version 15. Descriptive analyses were presented using mean \pm standard deviation (SD) for the variables. Levene test was used to assess the homogeneity of the variances. Chi-square $\left(\chi^{2}\right)$ or Fisher Exact test for ordinal and independent sample and $T$ test or Mann-Whitney $U$ test for scale were used to compare the variables be- tween patients with LP and controls, where appropriate. The capacity of $N / L$ ratio in predicting presence of the disease was analyzed using receiver operating characteristics (ROC) curve analysis. Correlation analysis was performed with Pearson or Spearmen according to homogeneity, where appropriate. When a significant cut-off value of N/L ratio was observed, the sensitivity, specificity, positive and negative predictive values were presented. The possible factors identified with univariate analyses were further entered into a multiple linear regression model which was used to identify independent predictors of $\mathrm{N} / \mathrm{L}$ ratio, with backward selection. The model fit was assessed using appropriate residual and goodness-of-fit statistics. A $p$-value of less than 0.05 was considered to show a statistically significant result.

\section{Results}

Fifty-five patients with LP (female $=28$, male $=27$ ) and 48 controls (female $=25$, male $=23$ ) were enrolled to the study. Median ages of the patient and control groups were 46 years (range: 18-80) and 43 years (range: 19-74), respectively. No statistical difference between patients group and control group was found. Most of the involvement sites of LP were upper and lower extremities and trunk. The generalized type was the most common type (54.5\%). Other clinical and laboratory findings in groups are given in Table $1 . \mathrm{N} / \mathrm{L}$ ratio was found statistically significant among groups (patient group; $2.5 \pm 1.1$ (1.2-7.3) vs. control group; $1.4 \pm 0.4(0.8-2.7), p<0.0001)$. The capacity of $\mathrm{N} / \mathrm{L}$ ratio above 1.42 was found significant in predicting presence of LP (area under curve (AUC) = 0.91, $95 \% \mathrm{Cl}$ : 0.85-0.96, $p<0.0001$ ) with $93 \%$ of sensitivity and $71 \%$ specificity. A moderate positive correlation was found between $N / L$ ratio-CRP, N/L ratio-ESR, and $\mathrm{N} / \mathrm{L}$ ratio-BSA (N/L ratio-CRP: $r=0.371, p=0.006 ; \mathrm{N} / \mathrm{L}$ ratio-ESR: $r=-0.40, p=0.003 ; \mathrm{N} / \mathrm{L}$ ratio-BSA; $r=0.43$, $p=0.01$ ) (Table 2). Involvement area $\left(\beta_{0}=2.1, \beta_{\mathrm{BSA}}=0.03\right.$, $p=0.001), \operatorname{CRP}\left(\beta_{0}=2.1, \beta_{\mathrm{CRP}}=0.05, p=0.006\right)$, and ESR $\left(\beta_{0}=1.8, \beta_{\mathrm{ESR}}=0.04, p=0.003\right)$ were identified as possible predictors of NLR, but only BSA and ESR were found as significant independent predictors in a multiple linear regression model $\left(\beta_{0}=1.4, \beta_{\mathrm{ESR}}: 0.04, p=0.003 ; \beta_{\mathrm{BSA}}=\right.$ 0.03, $p=0.002$ ).

\section{Discussion}

Lichen planus is a chronic inflammatory disease that affects the skin, genitalia, mucous membranes, and appendages [1, 7]. An immune-mediated mechanism may play a role in the pathogenesis of LP. Lichen planus is considered to be a T-cell mediated chronic inflammatory tissue reaction that results in a cytotoxic reaction against epithelial basal cells [8]. The pathogenesis of LP is related to upregulation of intercellular adhesion molecule-1 (ICAM-1) and Th1 immune response which is as- 


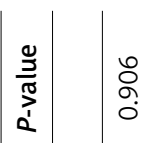

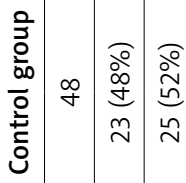

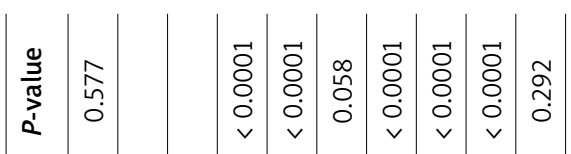

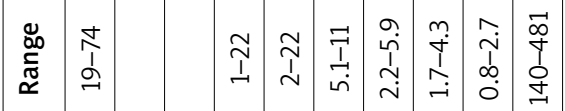

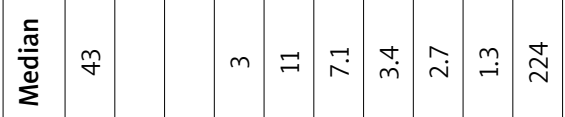

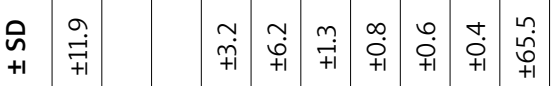

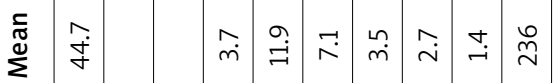

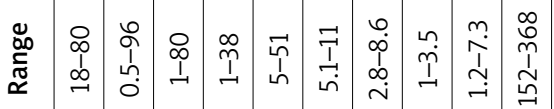

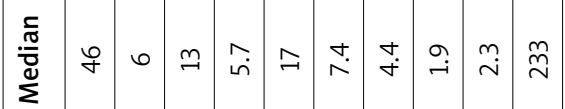

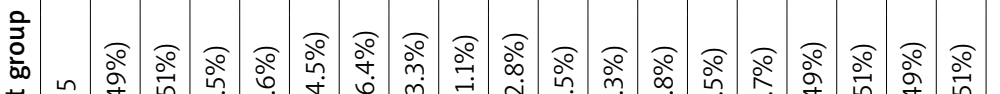

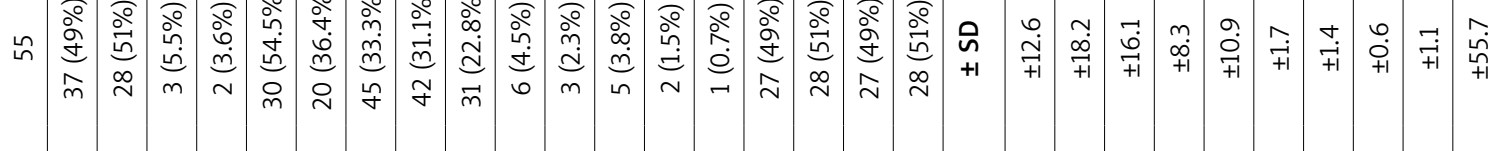

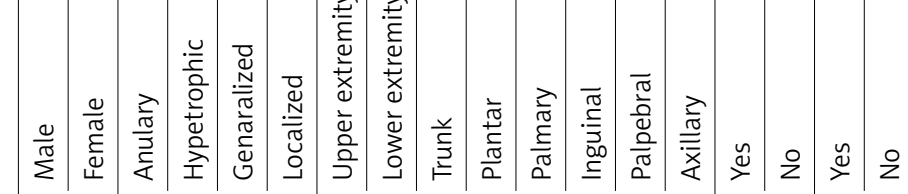

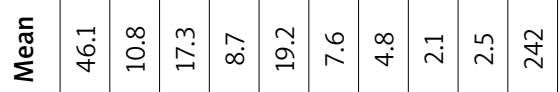


sociated with cytokines such as interferon (IFN)- $\gamma$, tumor necrosis factor (TNF)- $\alpha$, interleukin (IL)-1 $\alpha$, IL-6, and IL-8, IL-22, IL-17, IL-4 [9-13]. Toruniowa et al. found a significantly higher level of IL-6 in generalized cutaneous LP [14]. Immune reaction against an exogenous or endogenous antigen may be responsible at the onset of the disease by an aberrant immune response against to an intrinsic skin antigen. CD8+ T cells which are activated by antigens presented by keratinocytes, activated CD4+ helper $T$ cells and their cytokines produced chemokines that attract additional inflammatory cells, thereby promoting continued inflammation [8]. Upregulation of matrix metalloproteinase, proinflammatory mediators and proteases of mast cells, and toll-like receptors are other factors that have been proposed as contributors to LP [8, 15]. Lichen planus is assumed to be closely related to dyslipidemia and diabetes mellitus [1, 2]. In a case-control study, patients with lichen planus were found to have increased both metabolic and cardiovascular disorders in relation to controls most probably due to long standing inflammation [3].

Although there is an inflammatory process, etiology and pathogenesis of LP are not fully understood yet. Yamamoto et al. reported that both lymphocyte and neutrophil functions were impaired, and that cellular immunosuppression was a pathologic characteristic of oral lichen planus (OLP) [16]. Decreases in CD8+ T cells and NK activity were also reported in $\operatorname{OLP}[17,18]$. Lukac et al. suggested that OLP seemed to be associated with functional changes of salivary neutrophils involved in, reflecting the different pathophysiological mechanisms of the disease [19]. These findings support that LP is not only a chronic inflammatory disease restricted to the skin, but also a systemic one.

Noninvasive biomarkers of inflammation with a prognostic value such as CRP, ESR, WBC, procalcitonin, neopterin, interleukin (IL)-6, IL-8, interferon- $\alpha$, and TNF- $\alpha$ are widely used and have been evaluating for years to make accurate diagnosis, because the results of cultures, laboratory and radiologic evaluations may not always help the clinicians [20-22]. These markers can help us to show systemic inflammation; however, most of these markers are usually expensive and ineffective to use in clinical practice. Thus, there is still a need for new simple, specific, and inexpensive biomarkers for diagnosis and follow-up of inflammatory diseases such as LP. The other problem is a difficulty to reach some markers in the hospital laboratory. The need for new markers with high sensitivity and specificity is remaining. Normally, CRP and ESR increase owing to inflammation to the high level. High levels of CRP and ESR with high N/L ratio can support the systemic inflammatory process in LP. Moreover, BSA is significant on N/L ratio. Increase in BSA, CRP and ESR were related to an increase in $N / L$ ratio in our study. Our study showed that N/L ratio can be used for evaluating the inflammatory status and severity of disease in LP.
Table 2. Significant linear correlation analysis of some parameters in patients with lichen planus

\begin{tabular}{lcc}
\hline Parameters & $r$ & $P$-value \\
\hline N/L ratio-ESR & 0.40 & 0.003 \\
\hline N/L ratio-CRP & 0.37 & 0.006 \\
\hline N/L ratio-BSA & 0.43 & 0.01 \\
\hline ESR-CRP & 0.59 & $<0.0001$ \\
\hline ESR-age & 0.39 & 0.003 \\
\hline CRP-WBC & 0.48 & $<0.0001$ \\
\hline $\begin{array}{l}\text { ESR-WBC } \\
\text { Platelets-WBC }\end{array}$ & 0.51 & $<0.0001$ \\
\hline $\begin{array}{l}\text { Duration time-involvement } \\
\text { of mouth }\end{array}$ & 0.38 & 0.003 \\
\hline $\begin{array}{l}\text { Involvement of nail/involvement } \\
\text { of mouth }\end{array}$ & 0.29 & 0.03 \\
\hline ESR - erythrocyte sedimentation rate, BSA & 0.41 & 0.01 \\
\hline
\end{tabular}

ESR - erythrocyte sedimentation rate, BSA - body surface area, CRP $C$-reactive protein, $r$-correlation coefficient, $N / L$ - neutrophile-lymphocyte, WBC - white blood cells.

In addition, understanding the role of neutrophils could help to clarify the pathogenesis of LP.

Neutrophil lymphocyte ratio is a simple and inexpensive index of systemic inflammatory burden that correlates with prognosis in distinct disease conditions such as acute coronary syndrome, acute pancreatitis, and ulcerative colitis [23-25]. N/L ratio is considered as a systemic inflammatory marker. Because of this, systemic inflammation measured by N/L ratio has an association with prevalent chronic conditions [4]. N/L ratio was studied in various diseases including psoriasis and Behcet's disease. Relationship between severity of disease and increased inflammatory response are supported in these studies [5, 6]. Neutrophilia and lymphocytopenia is a physiological response of the innate immune system to systemic inflammation. A significant decrease in circulating lymphocyte count after systemic inflammation has been described [26]. The lymphocytopenia mechanism consist of margination of lymphocytes within the reticulo/endothelial system, liver and splanchnic lymphatic system and also redistribution of lymphocytes within the lymphatic system and marked accelerated apoptosis $[27,28]$. There is dense lymphocytic inflammation of the skin in LP, so margination of lymphocytes within the skin from the blood system may cause reduction in lymphocytes in our patients with LP. Neutrophilia is the opposite phenomenon during systemic inflammation as a result of demargination of neutrophils and stimulation of stem cells by growing factors (G-CSF). Zahorec et al. reported that under pathologic conditions such as severe infection or systemic inflammation, the $\mathrm{N} / \mathrm{L}$ ratio was increased and the severity of the clinical status and clinical out- 
come correlated well with the N/L ratio [29]. In our study, levels of WBC, neutrophil, N/L ratio, CRP, ESR except lymphocyte increased in patients with LP compared with controls. The rise in N/L ratio correlated with an increase in ESR, CRP, and BSA with high sensitivity and specificity. In addition, inflammatory parameters such as N/L ratio, WBC, CRP, ESR had a positive correlation with each other.

\section{Conclusions}

In this study we have shown that patients with LP have a higher $\mathrm{N} / \mathrm{L}$ ratio than controls. This might be associated with a systemic inflammatory process in LP. N/L ratio may be recommended for the estimation of disease severity and treatment option. Future studies should be performed in a large series of patients with LP and also before and after treatment. Moreover, new biomarkers should be investigated.

\section{Conflict of interest}

The authors declare no conflict of interest.

\section{References}

1. Arias-Santiago S, Buendia-Eisman A, Aneiros-Fernandez J, et al. Lipid levels in patients with lichen planus: a case-control study. J Eur Acad Dermatol Venereol 2011; 25: 1398-401.

2. Seyhan M, Ozcan H, Sahin I, et al. High prevalence of glucose metabolism disturbance in patients with lichen planus. Diabetes Res Clin Pract 2007; 77: 198-202.

3. Saleh N, Samir N, Megahed H, Farid E. Homocysteine and other cardiovascular risk factors in patients with lichen planus. J Eur Acad Dermatol Venereol 2014; 28: 1507-13.

4. Imtiaz F, Shafique K, Mirza SS, et al. Neutrophil lymphocyte ratio as a measure of systemic inflammation in prevalent chronic diseases in Asian population. Int Arch Med 2012; 26: 2.

5. Bulbul Sen B, Rifaioglu EN, Ekiz O, et al. Neutrophil to lymphocyte ratio as a measure of systemic inflammation in psoriasis. Cutan Ocul Toxicol 2014; 33: 223-7.

6. Ozturk C, Balta S, Balta I, et al. Neutrophil-lymphocyte ratio and carotid-intima media thickness in patients with Behcet disease without cardiovascular involvement. Angiology 2015; 66: 291-6.

7. Drogoszewska B, Chomik P, Polcyn A, Michcik A. Clinical diagnosis of oral erosive lichen planus by direct oral microscopy. Postep Derm Alergol 2014; 31: 222-8.

8. Roopashree MR, Gondhalekar RV, Shashikanth MC, et al. Pathogenesis of oral lichen planus: a review. J Oral Pathol Med 2010; 39: 729-34.

9. Lehman JS, Tollefson MM, Gibson LE. Lichen planus. Int J Dermatol 2009; 48: 682-94.

10. Chen X, Liu Z, Yue Q. The expression of TNF-alpha and ICAM-1 in lesions of lichen planus and its implication. J Huazhong Univ Sci Technology Med Sci 2007; 27: 739-41.

11. Carbone T, Nasorri F, Pennino D, et al. CD56highCd16-NK cell involvement in cutaneous lichen planus. Eur J Dermatol 2010; 20: 724-30.

12. Hussein MR. Evaluation of angiogenesis in normal and lichen planus skin by CD34 protein immunohistochemistry: preliminary findings. Cell Biol Int 2007; 31: 1292-5.
13. Rhodus NL, Cheng B, Ondrey F. Th1/Th2 cytokine ratio in tissue transudates from patients with oral lichen planus. Mediators Inflamm 2007; 2007: 19854.

14. Toruniowa B, Krasowska D, Koziol M, et al. Serum level of IL-6 in mycosis fungoides, psoriasis, and lichen planus. An NY Acad Sci 1995; 762: 432-4.

15. El Tawdy A, Rashed L. Downregulation of TLR-7 receptor in hepatic and non-hepatic patients with lichen planus. Int J Dermatol 2012; 51: 785-9.

16. Yamamoto T, Yoneda K, Ueta E, Osaki T. Cellular immunosuppression in oral lichen planus. J Oral Pathol Med 1990; 19: 464-70.

17. Simon M Jr, Keller J. Subpopulations of T lymphocytes in peripheral blood and in skin lesions in lichen ruber planus. Dermatologica 1984; 169: 112-6.

18. Hunyadi J, Simon M Jr, Kornacher J, Hornstein OP. Decreased natural killer cell activity in lichen ruber planus. Acta Derm Venereol 1986; 66: 435-8.

19. Lukac J, Mravak-Stipetic M, Knezevic M, et al. Phagocytic functions of salivary neutrophils in oral mucous membrane diseases. J Oral Pathol Med 2003; 32: 271-4.

20. Kocabas E, Sarikcioglu A, Aksaray N, et al. Role of procalcitonin, C-reactive protein, interleukin-6, interleukin-8 and tumor necrosis factor-alpha in the diagnosis of neonatal sepsis. Turk J Pediatr 2007; 49: 7-20.

21. Gendrel D, Raymond J, Coste J, et al. Comparison of procalcitonin with C-reactive protein, interleukin 6 and interferon-alpha for differentiation of bacterial vs. viral infections. Pediatr Infect Dis J 1999; 18: 875-81.

22. Prat C, Sancho JM, Dominguez, et al. Evaluation of procalcitonin, neopterin, C-reactive protein, IL-6 and IL-8 as a diagnostic marker of infection in patients with febrile neutropenia. Leuk Lymphoma 2008; 49: 1752-61.

23. Tamhane UU, Aneja S, Montgomery D, et al. Association between admission neutrophil to lymphocyte ratio and outcomes in patients with acute coronary syndrome. Am J Cardiol 2008; 102: 653-7.

24. Azab B, Jaglall N, Atallah JP, et al. Neutrophil-lymphocyte ratio as a predictor of adverse outcomes of acute pancreatitis. Pancreatology 2011; 11: 445-52.

25. Torun S, Tunc BD, Suvak B, et al. Assessment of neutrophillymphocyte ratio in ulcerative colitis: a promising marker in predicting disease severity. Clin Res Hepatol Gastroenterol 2012; 36: 491-7.

26. Jilma B, Blann A, Pernerstorfer T, et al. Regulation of adhesion molecules during human endotoxemia. No acute effects of aspirin. Am J Respir Crit Care Med 1999; 159: 857-63.

27. Ayala A, Herdon CD, Lehman DL, et al. Differential induction of apoptosis in lymphoid tissues during sepsis: variation in onset, frequency, and the nature of the mediators. Blood 1996; 87: 4261-75.

28. Hotchkiss RS, Swanson PE, Freeman BD, et al. Apoptotic cell death in patients with sepsis, shock, and multiple organ dysfunction. Crit Care Med 1999; 27: 1230-51.

29.Zahorec R. Ratio of neutrophil to lymphocyte counts - rapid and simple parameter of systemic inflammation and stress in critically ill. Bratisl Lek Listy 2001; 102: 5-14. 